\title{
A TOPOLOGICAL NOTE ON THE ADAMS SPECTRAL SEQUENCE BASED ON MORAVA'S $K$-THEORY
}

\author{
NOBUAKI YAGITA
}

Abstract. In this note, we compute the Hopf algebra $K(n)_{*}(K(n))$ and consider the Adams spectral sequence of $K(n)_{*}(-)$ theory.

1. Introduction. Miller and Ravenel [3] showed that

$$
\operatorname{Ext}_{\mathrm{BP}_{*} \mathrm{BP}}\left(\mathrm{BP}_{*},\left[v_{n}^{-1}\right] \mathrm{BP}_{*} / I_{n}\right) \cong \operatorname{Ext}_{K(n)} K(n)\left(K(n)_{*}, K(n)_{*}\right) \text {. }
$$

Miller, Morava, Ravenel and Wilson [3], [4], [5] make use of the right side group to compute stable homotopy groups of spheres. However, the group is not straightway induced by the Adams spectral sequence based on Morava's extraordinary $K(n)$-theory [5]; in fact $K(n)_{*} K(n)$ is purely algebraically defined and is not isomorphic to $\pi_{*}(K(n) \wedge K(n))=K(n)_{*}(K(n))$.

In this paper, however, we show that the group is isomorphic to $E_{2}$-term of the spectral sequence, i.e., if $V(n-1)$ exists,

$\operatorname{Ext}_{K(n)_{*} K(n)}\left(K(n)_{*}, K(n)_{*}\right) \cong \operatorname{Ext}_{K(n)_{*}(K(n))}\left(K(n)_{*}\left(S^{0}\right), K(n)_{*}(V(n-1))\right)$.

2. $\left[v_{m}^{-1}\right] \mathrm{BP}(S)_{*}(\mathrm{BP}(S))$. Let $\mathrm{BP}_{*}(-)$ be the Brown-Peterson homology theory at $a$ fixed odd prime $p$, with the coefficient $\mathrm{BP}_{*} \cong Z_{(p)}\left[v_{1}, \ldots\right]$. By using the Sullivan's bordism theory with singularities [9], we can construct the homology theory $\mathrm{BP}(S)_{*}(-)$ with the coefficient $\mathrm{BP}_{*} /(S)$ where $S=$ $\left(P_{1}, \ldots\right)$ is a regular sequence of $P_{i} \in \mathrm{BP}_{*}$. Since $\mathrm{BP}(S)$ is a ring spectrum [7], $\mathrm{BP}(S)_{*}(\mathrm{BP}(S))$ is a $\mathrm{BP}_{*}$-algebra. Note that $\mathrm{BP}_{*}(\mathrm{BP}) \cong \mathrm{BP}_{*}\left[t_{1}^{\prime}, \ldots\right]$ and $H_{*}\left(K Z_{p} ; Z_{p}\right) \cong Z_{p}\left[t_{1}^{\prime}, \ldots\right] \otimes \Lambda\left[\tau_{0}^{\prime}, \ldots\right]$ (see [1], [10]). Let $i_{1}: \mathrm{BP}_{*}(\mathrm{BP}) \rightarrow$ $\mathrm{BP}(S)_{*}(\mathrm{BP}) \rightarrow \mathrm{BP}(S)_{*}(\mathrm{BP}(S))$ and $i_{2}: \operatorname{BP}(S)_{*}(\mathrm{BP}(S)) \rightarrow H_{*}\left(\mathrm{BP}(S) ; Z_{p}\right) \rightarrow$ $H_{*}\left(K Z_{p} ; Z_{p}\right)$ be maps induced by natural inclusion maps $i_{1}: \mathrm{BP} \rightarrow \operatorname{BP}(S)$ and $i_{2}: \mathrm{BP}(S) \rightarrow K Z_{p}$.

THEOREM 1. Let $S=\left(v_{j_{0}}=p, v_{j_{1}}, \ldots\right)$ and $j_{k}<j_{k+1}$. If $j_{k}=k$ for $0 \leqslant k \leqslant$ $m-1$ and $j_{m} \neq m$, then we have a $\mathrm{BP}_{*}$-algebra isomorphism

$$
\begin{aligned}
{\left[v_{m}^{-1}\right] \mathrm{BP}(S)_{*} } & (\mathrm{BP}(S)) \\
& \cong\left[v_{m}^{-1}\right] \mathrm{BP}_{*}\left[t_{1}, \ldots\right] \otimes \Lambda\left[\tau_{0}, \ldots, \tau_{m-1}\right] /\left(S, \eta_{R}(S)\right)
\end{aligned}
$$

where $t_{i}, \tau_{i}$ are elemenis in $\mathrm{BP}(S)_{*}(\mathrm{BP}(S))$ so that $i_{1}\left(t_{i}^{\prime}\right)=t_{i}, i_{2}\left(\tau_{i}\right)=\tau_{i}^{\prime}$ and $\left(S, \eta_{R}(S)\right)$ is the ideal generated by $\left(v_{j_{0}}, \ldots, \eta_{R}\left(v_{j_{0}}\right), \ldots\right)$.

Received by the editors March 1, 1977 and, in revised form, September 6, 1977.

AMS (MOS) subject classifications (1970). Primary 55B20, 55H15; Secondary 55G25. 
Proof. Let $S_{n}=\left(v_{j_{0}}, \ldots, v_{j_{n}}\right)$. Since $\operatorname{BP}\left(S_{n}\right)$ is constructed by the cofiber map

$$
\mathrm{BP}\left(S_{n-1}\right) \underset{v_{j_{n}}}{\rightarrow} \mathrm{BP}\left(S_{n-1}\right) \rightarrow \mathrm{BP}\left(S_{n}\right)
$$

there is the $\mathrm{BP}_{*} / S$-module exact sequence

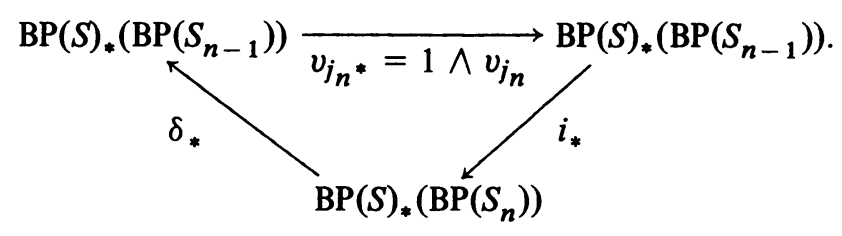

Here, we note that by using the Sullivan (Bockstein) exact sequence [7], [9], we can easily see that $\mathrm{BP}(S)_{*}(\mathrm{BP}) \cong \mathrm{BP}_{*} /(S) \otimes_{\mathrm{BP}_{*}} \mathrm{BP}_{*}(\mathrm{BP})$, since $\mathrm{BP}$ is torsion free and $S$ is regular.

Assume $n<m$. Then, in $\mathrm{BP}_{*}(\mathrm{BP}), 1 \wedge v_{j_{n}}=\eta_{R}\left(v_{j_{n}}\right)=0 \bmod$ $\left(p, \ldots, v_{m-1}\right) \subset(S)$. By induction and compatibility of the product, we can prove that $v_{j^{*}}=0$ in $\operatorname{BP}(S)_{*}\left(\operatorname{BP}\left(S_{n-1}\right)\right)$ (see the proof of Theorem 3.4 in [9]). And let $\tau_{n} \in \delta_{*}^{-1}(1)$; then we can prove

$$
\mathrm{BP}(S)_{*}\left(\mathrm{BP}\left(S_{n}\right)\right) \cong \mathrm{BP}_{*} /(S) \otimes_{\mathrm{BP}_{*}} \mathrm{BP}_{*}(\mathrm{BP}) \otimes \Lambda\left[\tau_{0}, \ldots, \tau_{n}\right],
$$

and moreover, we can choose $\tau_{i}$ so that $i_{3}\left(\tau_{i}\right)=\tau_{i}^{\prime}$ where $i_{3}: \operatorname{BP}(S)_{*}\left(\operatorname{BP}\left(S_{n}\right)\right)$ $\rightarrow H_{*}\left(K Z_{p} ; Z_{p}\right)$ is the map induced by the natural inclusion map $i_{3}: \operatorname{BP}\left(S_{n}\right)$ $=\mathrm{BP}\left(p, \ldots, v_{j_{n}}\right) \rightarrow K Z_{p}$ (see [9]).

Assume $n \geqslant m$. By [6] in $\mathrm{BP}_{*}(\mathrm{BP})$ we have

$$
\begin{aligned}
1 \wedge v_{j_{n}}=\eta_{R}\left(v_{j_{n}}\right)= & v_{m} t_{j}^{p^{m}}-v_{m}^{p^{\prime} t_{j}^{\prime}} \\
& \quad \bmod \left(p, \ldots, \hat{v}_{m}, \ldots, \eta_{R}\left(v_{m+1}\right), \ldots, \eta_{R}\left(v_{j_{n}-1}\right)\right) \\
& \subset\left(p, \ldots, \hat{v}_{m}, \ldots, t_{1}^{\prime}, \ldots, t_{j-1}^{\prime}\right) .
\end{aligned}
$$

where $j=j_{n}-m$. Therefore $\eta_{R}\left(v_{j_{n}}\right)$ is a non zero divisor of $\left[v_{m}^{-1}\right] \mathrm{BP}_{*}(\mathrm{BP}) /\left(S, \eta_{R}\left(S_{n-1}\right)\right)$. By induction and also the compatibility of the product, we can prove that $v_{j_{n}}$ is injective in $\left[v_{m}^{-1}\right] \mathrm{BP}(S)_{*}\left(\operatorname{BP}\left(S_{n-1}\right)\right)$, therefore the proof is completed. have

Remark 1. By the exact functor theorem for $\mathrm{BP}_{*} /\left(p, \ldots v_{m-1}\right)[11]$ we

$$
\left[v_{m}^{-1}\right] \mathrm{BP}(S)_{*}(-) \cong\left[v_{m}^{-1}\right] \mathrm{BP}_{*} / S \otimes_{\mathrm{BP}_{*}} \mathrm{BP}\left(p, \ldots, v_{m-1}\right)_{*}(-) .
$$

Using this fact, we can also prove Theorem 1.

By arguments similar to the proof of Theorem 1, we can prove that

$$
\begin{aligned}
{\left[v_{m}^{-1}\right] \mathrm{BP}(S)_{*} } & (\mathrm{BP}(S) \wedge \mathrm{BP}(S)) \\
& \cong\left[v_{m}^{-1}\right] \mathrm{BP}(S)_{*}(\mathrm{BP}(S)) \bigotimes_{\mathrm{BP}_{*}} \mathrm{BP}(S)_{*}(\mathrm{BP}(S)) .
\end{aligned}
$$


Hence $\left[v_{m}^{-1}\right] \mathrm{BP}(S)_{*}(\mathrm{BP}(S))$ is a Hopf algebra in the standard way [1].

THEOREM 2. Let $\psi$ be the coaction map in $\left[v_{m}^{-1}\right] \mathrm{BP}(S)_{*}(\mathrm{BP}(S))$.

(1) $\Sigma^{\mu} \psi\left(t_{k}\right)=\Sigma^{\mu} t_{i} \otimes t_{k-i}^{p^{i}}$ where $\mu$ is the product of the formal group law of $\mathrm{BP}_{*}$.

(2) $\psi\left(\tau_{k}\right)=\Sigma_{i>0} \tau_{i} \otimes t_{k-i}^{i}+1 \otimes \tau_{k}$.

Proof. By the coaction of $\mathrm{BP}_{*}(\mathrm{BP})$ [1], [6] and the compatibility of the product, (1) is immediate. Let $i_{4}: \mathrm{BP}\left(S_{m-1}\right)_{*}\left(\mathrm{BP}\left(S_{m-1}\right)\right) \rightarrow \mathrm{BP}(S)_{*}(\mathrm{BP}(S))$ be the natural inclusion map. Since $\left[v_{m}^{-1}\right] i_{4}$ is epic by Theorem 1 , we may prove that (2) holds in $\operatorname{BP}\left(S_{m-1}\right)_{*}\left(\operatorname{BP}\left(S_{m-1}\right)\right)$. Let $i_{5}: \operatorname{BP}\left(S_{m-1}\right)_{*}\left(\mathrm{BP}\left(S_{m-1}\right)\right) \rightarrow$ $H_{*}\left(K Z_{p} ; Z_{p}\right)$ be the natural inclusion map. Then by Theorem 3 in [10], we have $i_{5}\left(\psi\left(\tau_{k}\right)-(2)\right)=0$. But $\operatorname{ker} i_{5}=\operatorname{Ideal}\left(v_{m}, v_{m+1}, \ldots\right)$. Since $\operatorname{dim} \tau_{k}=$ $2 p^{k}-1<2\left(p^{m}-1\right)$, we have $\psi\left(\tau_{k}\right)=(2)$.

Example 1. Denote $\operatorname{BP}\left(p, \ldots, v_{n-1}\right)$ by $P(n)$ and $\operatorname{BP}\left(p, \ldots, \hat{v}_{n}, \ldots\right)$ by $k(n)$ due to notations in [2] $[4]$.

$$
\begin{aligned}
P(n)_{*}(P(n)) \cong P(n)_{*} & \otimes_{\mathrm{BP}_{*}} \mathrm{BP}_{*}(\mathrm{BP}) \otimes \Lambda\left[\tau_{0}, \ldots, \tau_{n-1}\right], \\
{\left[v_{n}^{-1}\right] k(n)_{*}(k(n)) \cong } & {\left[v_{n}^{-1}\right] k(n)_{*} \otimes_{\mathrm{BP}_{*}} \mathrm{BP}_{*}(\mathrm{BP}) } \\
& \otimes \Lambda\left[\tau_{0}, \ldots, \tau_{n-1}\right] /\left(v_{n} t_{i}^{p^{n}}-v_{n}^{p^{i}} t_{i}\right) .
\end{aligned}
$$

3. $\operatorname{Ext}_{K(n)_{*}(K(n))}\left(K(n)_{*}\left(S^{0}\right), \quad K(n)_{*}(V(n-1))\right)$. Let $K(n)_{*}(-)=$ $\left[v_{n}^{-1}\right] k(n)_{*}(-)[2],[3],[4]$. Then we have

$$
\begin{aligned}
K(n)_{*}(K(n)) & =\left[v_{n}^{-1}\right] k(n)_{*}(K(n)) \cong\left[v_{n}^{-1}\right] c\left(K(n)_{*}(k(n))\right) \\
& \cong\left[v_{n}^{-1}\right]\left[\eta_{R}\left(v_{n}\right)^{-1}\right] c\left(k(n)_{*}(k(n))\right) \cong\left[v_{n}^{-1}\right] k(n)_{*}(k(n))
\end{aligned}
$$

where $c$ is the conjugation map.

On the other hand, in [3], [4], [6], $K(n)_{*} K(n)$ (without parentheses) is defined by

$$
K(n)_{*} K(n)=K(n)_{*} \otimes_{\mathrm{BP}_{*}} \mathrm{BP}_{*}(\mathrm{BP}) /\left(v_{n} t_{i}^{p^{n}}-v_{n}^{p^{i}} t_{i}\right)
$$

Hence we have

$$
K(n)_{*}(K(n)) \cong K(n)_{*} K(n) \otimes \Lambda\left[\tau_{0}, \ldots, \tau_{n-1}\right] .
$$

LEMMA 1. Let $L, R$ be $K(n)_{*} K(n)$-comodules; then we have

$$
\operatorname{Ext}_{K(n)_{*} K(n)}(L, R) \cong \operatorname{Ext}_{K(n)_{*}(K(n))}\left(L, R \otimes \Lambda\left[\tau_{0}, \ldots, \tau_{n-1}\right]\right) .
$$

Proof. Let $0 \rightarrow R \rightarrow R_{1} \rightarrow \ldots$ be the $K(n)_{*} K(n)$-cobar resolution. Then each $R_{i}$ is an extended $K(n)_{*} K(n)$-comodule. Then

$$
0 \rightarrow R \otimes \Lambda\left[\tau_{0}, \ldots, \tau_{n-1}\right] \rightarrow R_{1} \otimes \Lambda\left[\tau_{0}, \ldots, \tau_{n-1}\right] \rightarrow \ldots
$$

is a resolution so that each $R_{i} \otimes \Lambda\left[\tau_{0}, \ldots, \tau_{n-1}\right]$ is an extended $K(n)_{*}(K(n))$-comodule. Hence we need only prove that 


$$
\operatorname{Hom}_{K(n)_{*} K(n)}\left(L, R_{i}\right) \cong \operatorname{Hom}_{K(n)_{*}(K(n))}\left(L, R_{i} \otimes \Lambda\left[\tau_{0}, \ldots, \tau_{n-1}\right]\right),
$$

where Hom is the homomorphisms of comodules.

Let $f \in$ (the right side of (1)) and $a \in L$. Let $f(a)=\Sigma b_{\alpha} \tau^{\alpha}$ where $b_{\alpha} \in R_{i}$

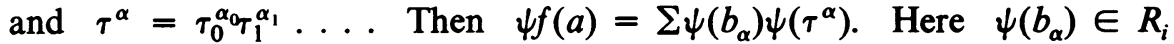
$\otimes_{K(n)_{*}} K(n)_{*} K(n)$ and $\psi\left(\tau^{\alpha}\right)=1 \otimes \tau^{\alpha}+\Sigma_{\left|\alpha^{\prime}\right|<|\alpha|} \tau^{\beta} \otimes t^{\gamma} \tau^{\alpha^{\prime}}$. But $\psi(f(a))=$ $f(\psi(a)) \in R_{i} \otimes_{K(n)_{*}} K(n)_{*} K(n)$. Hence if $|\alpha|>0$ then $\psi\left(b_{\alpha}\right)=0$, so $b_{\alpha}=0$. Therefore we can consider $f \in$ (the left side of (1)).

Now consider the Adams spectral sequence (see $\$ 15$ in [1]). Since $K(n)_{*}(K(n))$ satisfies assumptions (i), (ii) on p. 317 in [1], there exists a spectral sequence whose $E_{2}$-term is

$$
\operatorname{Ext}_{K(n)_{*}(K(n))}\left(K(n)_{*}(X), K(n)_{*}(Y)\right)
$$

for arbitrary spectra $X, Y$. However, the assumption (iii) is not satisfied; the spectral sequence doesn't necessarily converge to $\pi_{*}[X, Y]_{(p)}$.

Here, recall that $V(n-1)$ is the spectrum such that $\mathrm{BP}_{*}(V(n-1)) \cong$ $\mathrm{BP}_{*} /\left(p, \ldots, v_{n-1}\right)[8]$. Miller-Ravenel [3] showed

$$
\operatorname{Ext}_{K(n)_{*} K(n)}\left(K(n)_{*}, K(n)_{*}\right) \cong \operatorname{Ext}_{\mathrm{BP}_{*}(\mathrm{BP})}\left(\mathrm{BP}_{*},\left[v_{n}^{-1}\right] \mathrm{BP}_{*} /\left(p, \ldots, v_{n-1}\right)\right)
$$

We have the following theorem.

THEOREM 3. If $V(n-1)$ exists,

$$
\begin{aligned}
& \operatorname{Ext}_{K(n)_{*} K(n)}\left(K(n)_{*}, K(n)_{*}\right) \\
& \cong \operatorname{Ext}_{K(n)_{*}(K(n))}\left(K(n)_{*}\left(S^{0}\right), K(n)_{*}(V(n-1))\right) .
\end{aligned}
$$

Proof. By Lemma 1, we need only prove that there is a $K(n)_{*}(K(n))$ comodule isomorphism

$$
K(n)_{*}(V(n-1)) \cong K(n)_{*} \otimes \Lambda\left[\tau_{0}, \ldots, \tau_{n-1}\right]
$$

If $V(n-1)$ exists, then for $0 \leqslant i \leqslant n-1$, there exists $V(i)$ and it is constructed by the following cofibering

$$
V(i-1) \underset{v_{i}}{\rightarrow} V(i-1) \rightarrow V(i) .
$$

By arguments similar to the proof of Theorem 1, the proof is easy.

REMARK 2. In $k(n)_{*}(-)$ theory, assumptions (i), (iii) are satisfied, but (ii) is not. Hence, for connected spectra $X, Y$, there exists the spectral sequence $E_{i}$ which converges to $\pi_{*}[X, Y]_{(p)}$. However, the $E_{2}$-term seems to be complicated.

\section{REFERENCES}

1. J. F. Adams, Stable homotopy and generalised homology, Univ. Chicago Press, Chicago, Ill., 1974.

2. D. C. Johnson and W. S. Wilson, BP operations and Morava's extraordinary $K$-theories, Math. Z. 144 (1975), 55-75. 
3. H. R. Miller and D. C. Ravenel, Morava stabilizer algebras and the localization of Novikov's $E_{2}$-term, Duke Math. J. 44 (1977), 433-447.

4. H. R. Miller, D. C. Ravenel and W. S. Wilson, Novikov's Ext $^{2}$ and the nontriviality of the gamma family, Bull. Amer. Math. Soc. 81 (1975), 1073-1075.

5. J. Morava, Structure theorems for cobordism comodules (to appear).

6. D. C. Ravenel, The structure of Morava stabilizer algebras, Invent. Math. 37 (1976), 109-120.

7. N. Shimada and N. Yagita, Multiplications in the complex bordism theory with singularities, Publ. RIMS, Kyoto Univ. 12 (1976), 259-293.

8. L. Smith, On realizing complex cobordism modules, Amer. J. Math. 92 (1970), 793-856.

9. N. Yagita, On the algebraic structure of cobordism operations with singularities, J. London Math. Soc. 16 (1977), 131-141.

10. J. Milnor, The Steenrod algebra and its dual, Ann. of Math. (2) 67 (1958), 250-271.

11. N. Yagita, The exact functor theorem for BP ${ }_{*} / I_{n}$-theory, Proc. Japan Acad. 52 (1976), 1-3.

Department of Mathematics, Tokyo Institute of Technology, Oh-okayama Meguroku, TOKYO, JAPAN 